\title{
Enhanced Macroporous Cationic Chitosan Hydrogel by Freezing and Thawing Method with Superadsorption Capacity for Anionic Dyes
}

\section{Zahraa H. Athab}

University of Babylon

Ahmed F. Halbus ( $\square$ ahmed.halbus@uobabylon.edu.iq )

University of Babylon https://orcid.org/0000-0001-9060-7073

Ahmed S. Abbas

University of Babylon

Jasim M. Salman

University of Babylon

Abbas J. Atiyah

University of Babylon

\section{Research Article}

Keywords: Interconnected porous chitosan hydrogel, Monolith, Freezing and thawing, Adsorption, recyclability.

Posted Date: February 4th, 2022

DOI: https://doi.org/10.21203/rs.3.rs-1313836/v1

License: (c) (i) This work is licensed under a Creative Commons Attribution 4.0 International License.

Read Full License 


\section{Abstract}

In this work, we have developed a simple method to prepare cationic chitosan hydrogel with interconnected porous structure using freeze-thaw process and the obtained hydrogel was named FCS hydrogel. Scanning electron microscopy (SEM) imaging revealed that the synthesized hydrogel demonstrated interconnected porous structure in the range of 5-20 $\mu \mathrm{m}$. We also showed that the FCS hydrogel exhibits $\mathrm{pH}$ responsiveness behavior, and demonstrated reversible swelling and de-swelling behaviors maintaining their mechanical stability. We demonstrate that the FCS hydrogel swelling capacity decreased at alkaline $\mathrm{pH}$ and increased with a decrease in the $\mathrm{pH}$ value. Besides, the FCS hydrogel presented specific surface area of $78.25 \pm 8.75 \mathrm{~m}^{2} \mathrm{~g}^{-1}$, due to the cryogenic treatment of glutaraldehyde cross-linked chitosan hydrogel could increase the surface area and permeability of composite hydrogel and then strongly increasing the adsorption capacity. Subsequently, the FCS monolithic hydrogel tested dyes removal, which provides a high removal efficiency towards anionic dyes including congo red (CR) and sodium fluorescein (SFL) dyes. Significantly, we show that the FCS hydrogel could be regenerated and reused as an adsorbent for wastewater treatment without significant loss of pollutants removal efficiency over a number of adsorption and washing cycles. This study offers a promising environmental friendly and sustainable interconnected porous hydrogel for anionic dye removal from wastewater.

\section{Introduction}

Nowadays, water pollution caused by toxic organic materials are a considerable serious threat to the environment [1,2]. Dyes are the main source of wastewater toxicity in different industrial activities such as paper, textile, plastics, food and pharmaceutical industries [3]. Dye wastewater have harmful effect to the human and animal health and all the natural ecosystems due to these materials contain many aromatic compounds, which are chemically stable and highly toxic $[4,5]$. Therefore, developing new strategies and materials for removing these pollutants from wastewater has attracted important attention. Various techniques such as ion exchange, chemical precipitation, solid phase extraction, separation, biological treatment, and adsorption have been used for wastewater treatment [6-8]. Among these technologies, adsorption is considered the most effective, versatile and promising method for dyes removal owing to the flexible operation, convenient processing, cost-effective, and high removal efficiency [9]. Activated carbon has been a common adsorbent material, being used for removing many types of dyes [10-14]. However, sometimes the preparation of activated carbon is comparatively expensive and the separation and regeneration from treated water is complicated, which limited its application for water purification and thus increases requirements for substitutes adsorbent materials being economical and sustainable [15]. Hydrogels are considered novel materials used as adsorbents with rapid removing of dyes owing to their unique three-dimensional porous network structure, which can absorb large amounts of water with lack of solubility in water due to the chemical or physical crosslinking between the polymeric chains $[16,17]$. Furthermore, hydrogels materials provide hydrophilic function groups that can be acted as an active site for organic contaminations removal [18]. In addition, the hydrogel simply 
separates from treated solution and have the ability to recover and reuse a number of times, which meets the demand for sustainability and eco-friendly development [19]. Some types of hydrogels are named smart materials, which often respond to the stimulus of the surrounding environment, such as $\mathrm{pH}$, temperature, light, and magnetic and electric field owing to their functional groups presented along the polymer chains [20]. For instance, the swelling and deswelling behavior of these materials may be sensitive to any change in the $\mathrm{pH}$, temperature of the solution or even to the presence of the light [21]. Polysaccharides are desired candidates for preparing hydrogels material as adsorbents for wastewater treatment due to they are biodegradable, non-toxic and available in nature [22]. In particular, chitosan is a widely used biopolymer for preparing hydrogel for pollution remediation owing to its the second most abundant biopolymer in nature after cellulose and have high adsorption capacity due to containing a large number of functions group such as hydroxyl and amine groups, which make them efficient adsorbent for different organic pollutants removal $[23,24]$. Chitosan considerable to be an excellent example of a pH-responsive natural polymer [25]. Moreover, it has been found that chitosan hydrogel has a better adsorption capacity than that chitosan polymer with non-crosslinking specially in acidic medium due to chitosan can be dissolved in acidic environment [26]. Chitosan hydrogels designed via either chemical or physical cross-linking using different crosslinker agents such as glutaraldehyde, glyoxal, formaldehyde, ethylene glycol diglycidyl ether, epichlorohydrin and oxalic acid were reported [27-30]. The mechanical properties and the chemical stability of chitosan are improved after cross-linking process [31]. However, unmodified chitosan hydrogel has a low surface area and a lake of porosity, which resulting low adsorption capacities [30]. To overcome these restrictions, there are different physical and chemical methods have been used for modifying chitosan hydrogel in order to increase the surface area and to create desirable porous structure and improve the mechanical properties, which are very important in our application [32,33]. Chitosan has been chemically modified by grafting with nanoparticles or crosslinking with other polymers. Ehsan and co-workers have grafted chitosan with polyacrylamide matrix and dispersed $\mathrm{TiO}_{2}$ nanoparticles in the network structure $\left(\mathrm{TiO}_{2}\right.$-PAM-CS) using in situ technique. This composite material was applied for the adsorption of Sirius yellow K-CF dye from aqueous solution and it was showed high removal efficiency of this anionic dye [3]. Hu Tu et al. enhanced the chitosan hydrogel by constructing chitosan with different ratios of cellulose by using the environmentally friendly method and materials. The prepared hydrogels were not only low cost, but also possessed high mechanical properties. Besides, the hydrogels had outstanding high adsorption ability towards anionic dyes in aqueous solution and preformed a surface area $33.9 \mathrm{~m}^{2} \mathrm{~g}^{-1}$ when the ratio between chitosan: cellulose was 9:1 [34]. Mahmoodi et al. have studied the use of GO for modifation of chitosan hydrogel (GO-CTS) and amine graphene oxide-chitosan (AGO-CTS) hydrogel which boosting the adsorption capacity. The removal of diclofenac GO-CTS hydrogel was investigated and it was exhibited high adsorption efficiency with maximum adsorption capacity reach to $132.11 \mathrm{mg} \mathrm{g}^{-1}$ [35]. Alves D.C.S. et al. synthesised chitosan hydrogel modified with carbon nanotubes (HyCNT) and tested it adsorption efficiency towards phenol. They showed that HyCNT demonstrated high specific surface area, which is interesting characteristics for adsorption application [36]. However, these chemical modifications for chitosan hydrogel suffer from a number of disadvantages including synthesis these materials can be costly and time consuming [37]. In addition, the regeneration of these modified materials for adsorption 
application were very complicated [38]. Besides, added another polymer with different function groups led to a lack the selectivity in the adsorption process. Furthermore, there are added concerns over the environmental impact of CNT and GO, which if leached from the chitosan composites, can enter the aquatic system and have adverse effects on the aquatic ecosystem [39]. Moreover, GO and CNT particles may be blocking the pores of chitosan hydrogel, which is impact on the diffusion of the pollutants through the porous structure and led to decrease the adsorption capacity [40]. Therefore, various researches have been used physical modifications of hydrogels to control the porosity to form superporous structure with high swelling properties such as gas blowing or freeze drying techniques, which can increase its adsorption capacity [41-43]. The freeze-drying technique has its own disadvantage as well such as high energy required, time consuming and may led to shrinkage the network structure because the interfacial tension resulted by solvent evaporation thus limiting their usage $[44,45]$. This work provides new ideas for physical modification of chitosan hydrogel was used. Freezing and thawing method have been carried out on glutaraldehyde cross-linked chitosan hydrogel in order to produce porous chitosan hydrogel to increase the diffusion properties. It is worth noting that freeze-thaw process preferred because it provides open the pores in the structure of the CS matrix thus penetration from the external surface of the sorbent to the intraparticle pores and adsorption on both the external and internal available surfaces via interactions of the solute. Therefore, increase of the specific surface area due to the expansion of the chitosan network structure by changing the pores from close or blind pores to interconnected pores, which are desirable in wastewater treatment field. This is shown schematically in Fig. 1A,B. To the best of our knowledge, since there have no studies about use this type of modification for chitosan hydrogel by freezing-thawing treatment and used it for adsorption of anionic dyes.

\section{Materials And Methods}

\section{Materials}

Chitosan was obtained from across organics with a medium molecular weight (Mwt 100000-300000). Glutaraldehyde (50\%) was purchased from Sigma-Aldrich. Congo red, Methylene blue dye (MB) and Fluorescein dye (acid yellow 73) (FL) were also purchased from Sigma-Aldrich. Rhodamine (6G) was obtained from Chem. Supply. Acetic acid was purchased from macron fine chemicals. All other chemicals used in this research were used directly without further purification. All solutions in this study were prepared with distilled water.

\section{Preparation of chitosan hydrogel}

The chemically cross-linked chitosan hydrogel was prepared based on a modified version of the method described by Xinhong Chang et.al. using glutaraldihyde as cross linker agent [27]. $1.0 \mathrm{~g}$ of chitosan was dissolved in $50.0 \mathrm{~mL}$ of $1.0 \mathrm{vol} \%$ acetic acid solution under magnetic stirring at room temperature (solution 1), and $1.0 \mathrm{~mL}$ glutaraldehyde (50 wt \%) was dissolved at room temperature in $20.0 \mathrm{~mL}$ distilled water (solution 2). The two solutions were mixed at room temperature under vigorous stirring for 2 min and after that immediately placed into the plastic syringes of an internal diameter of $1 \mathrm{~cm}$ then gradually 
transformed into wet gels within 10 min and were aged for $24 \mathrm{~h}$. To form a porous chitosan hydrogel for wastewater treatment, the obtained chitosan hydrogels were frozen in a freezer at $-20^{\circ} \mathrm{C}$ for $48 \mathrm{~h}$ and then melted at room temperature. After cooling down the frozen chitosan hydrogel was washed with 0.01 $M$ solution of hydrochloric and then with distilled water to remove all unreacted material. Finally, the obtained porous monolithic chitosan hydrogel was named FCS and it was stored in distilled water (DW) at room temperature until use.

\section{Characterization}

The structural morphology of the fabricated chitosan hydrogel was performed by using scanning electron microscopy (JEOL JSM-6480 LV SEM instrument). The sample was fractured to create a fresh surface, then adhered onto an SEM stub. The sample was then sputter coated with gold via sputter coater and then transferred into the SEM chamber. The analysis of the functional groups of the powder chitosan and the synthesised chemical cross-linked chitosan were carried out via examined using Thermo Scientific Nicolet 380 FT-IR (Thermo Scientific, Hemel Hempstead, UK), supplied with attenuated total reflectance (ATR). Each sample was analyzed in the wavenumber range of $4000-500 \mathrm{~cm}^{-1}$ using 4 scans. The specific surface area, pore size distribution and total pore volume for the freeze dried chitosan hydrogel material was measured by using Micromeritics Tristar II 3000 (USA) BET- $\mathrm{N}_{2}$ surface area analyser using $\mathrm{N}_{2}$ gas adsorption analysis by stabilising at $-196^{\circ} \mathrm{C}$. Chitosan hydrogel was freeze dried using (VirTis SP SCIENTIFIC) to obtain the monolithic chitosan aerogel material and then was dried at $100{ }^{\circ} \mathrm{C}$ for 12 hours for degassing the samples before carrying out the surface area analysis. The pore size distributions were calculated using the Barett-Joyner-Halenda (BJH) model. A Zetasizer Nano instrument (Malvern Instruments, Malvern, UK) was used to measure the zeta potential on the surface of the FD-chitosan hydrogel. Chitosan hydrogel porosity percent $(\mathrm{P} \%)$ were calculated from the density of wet FCS hydrogel ( $\left.\rho_{\text {FCS wet }}\right)$ and the density of dry FCS hydrogel $\left(\rho_{\text {FCS dry }}\right)$. The swelling degree (SD) of FCS hydrogel was measured by using gravimetric analysis, from $\mathrm{pH} 2$ to 10 . Before measurement, the hydrogels were left in DW for $24 \mathrm{~h}$. They were then transferred in a water at the desired $\mathrm{pH}$ for $24 \mathrm{~h}$. The excess water on the surface of the sample was gently removed using filter paper before measurement. The weight of the hydrogel at a given $\mathrm{pH}(\mathrm{Ws})$ and the weight of the dehydrated hydrogel at $\mathrm{pH} 10(\mathrm{Wd})$ were recorded and used to calculate SR according to the following equation:

$\mathrm{SD}=\mathrm{Ws} / \mathrm{Wd}$

The swelling kinetics and the water uptake ratio (WU) of FCS hydrogel were also measured by gravimetry analysis. Briefly, the hydrogels were first plunged in $\mathrm{pH} 2$ solution, brought to equilibrium, and weighed $(\mathrm{Wg})$. They were then plunged in $\mathrm{pH} 10$ solution for 24 hours $(\mathrm{Wg})$, until equilibrium in the dehydrated state was reached. They were then immersed again in $\mathrm{pH} 2$ and then the weight of the hydrogels was recorded $(\mathrm{Wt})$ at different time through one hour. As mentioned previously, before weighing the excess of water on the surface of the hydrogels were absorbed with filter paper. The water uptake ratio WU is calculated using the following equation: 
$\mathrm{WU}=(\mathrm{Wt} / \mathrm{Wg}) \times 100$

The deswelling kinetics and the water retention ratio (WR) of the FCS hydrogel were also studied by gravimetry analysis. The FCS hydrogels, initially stored in distilled water at $\mathrm{pH} 2$ for $24 \mathrm{~h}$ then the weight of the hydrogels was recorded $(\mathrm{Wg})$, were then transferred in $\mathrm{pH} 10$ solution. Then, the weight of the hydrogels was recorded (Wt) at the same predetermined time intervals for swelling study. As mentioned above, before measuring the weight the excess of water on the surface of the hydrogels were absorbed carefully with filter paper. The water retention ratio WR was calculated using the following equation:

$\mathrm{WR}=(\mathrm{Wt} / \mathrm{Wg}) \times 100$

The zeta potential of FCS hydrogel was evaluated by dynamic light scattering (DLS) using Zetasizer Nano ZL instrument (Malvern, UK). FCS hydrogel was crashed into small parts and then dispersed in deionized water using an ultrasonic probe (Branson LTD). The measurement was carried out in triplicate at room temperature.

\section{Adsorption studies}

$\mathrm{CR}, \mathrm{MB}, \mathrm{R} 6 \mathrm{G}$ and $\mathrm{FL}$ were selected to examine the ability of adsorption and removal capacity of the chemical cross-linked CS hydrogel. The experimental data were recorded using a UV-Vis spectrophotometer at $498 \mathrm{~nm}, 664 \mathrm{~nm}, 525 \mathrm{~nm}$, and $490 \mathrm{~nm}$ for CR, MB, R6G and FL respectively. The prepared FCS hydrogels were put into $100 \mathrm{mg} / \mathrm{L}$ dye solutions. Then, the solutions were shaken in the dark condition regularly for 3 hours using a platform shaker (Gemmy orbit shaker model: VRN-480). The absorbance at the maximum absorption wavelength was measured before and after adsorption. The batch adsorption tests were carried out to evaluate the percentage of dye removal $(R \%)$ and the adsorption uptake quantity $\left(Q_{e}\right)$ of CR or SFL on FCS hydrogel whereby the monolithic hydrogel $(\sim 0.05 \mathrm{~g}$ of FCS, $3 \mathrm{~mL}$ volume) were plunged into isolated conical flasks with $100 \mathrm{~mL}$ serial solutions of $\mathrm{CR}$ or $\mathrm{SFL}$ dyes (10-60 $\left.\mathrm{mg} \mathrm{L}^{-1}\right)$. The samples were gently shaken in the dark condition at room temperature $(25 \pm 2$ ${ }^{\circ} \mathrm{C}$ ) in the dark condition with a shaker speed of $100 \mathrm{rpm}$ until reached the equilibrium. All experiments were carried out in triplicate and the concentration of anionic dyes solutions before and after the adsorption process were calculated using double beam UV-Vis spectrometry (1650 PC-UV-Visible spectrometer Shimadzu.) at the maximum absorbance of CR dye $(498 \mathrm{~nm})$ and FL dye $(490 \mathrm{~nm})$. The dye removal percentage $(\mathrm{R} \%)$ of the dyes and the adsorption quantity of the dye adsorbed per unit mass of adsorbent at particular time $Q_{t}$ and at equilibrium $Q_{e}\left(\mathrm{mg} \mathrm{g}^{-1}\right)$ were calculated according to the following equations: 


$$
\begin{gathered}
\% R=\frac{C_{0}-C_{t}}{C_{0}} \times 100 \% \\
Q_{t}=\frac{\left(C_{0}-C_{t}\right) V}{m} \\
Q_{e}=\frac{\left(C_{0}-C_{e}\right) V}{m}
\end{gathered}
$$

Where $C_{0}$ and $C_{t}$ are the initial dye concentration $\left(\mathrm{mg} \cdot \mathrm{L}^{-1}\right)$ before adsorption and is the concentration of dyes after adsorption at a given time t. $C_{e}\left(\mathrm{mg} \mathrm{L}^{-1}\right)$ is the concentration at equilibrium, $\mathrm{m}$ and $\mathrm{V}$ are the dose of FCS hydrogel $(\mathrm{g})$ and the volume of dye solution $(\mathrm{L})$.

\section{Adsorption isotherm}

The adsorption isotherms were investigated using both Langmuir and Freundlich isotherms for adsorption of CR and FL dyes on the FCS hydrogel. The Freundlich model is an empirical model and more applicable at low adsorbate concentrations. In the theoretical Freundlich model, it is assumed that adsorption of dye occurs on a heterogeneous surface of the adsorbent, it suggests multilayer adsorption and there is an interaction between the adsorbate and adsorbent molecules depend on the electrostatic interaction between negative and positive charges. The Freundlich isotherm parameters were calculated according the following equation:

$$
\log Q_{e}=\log K_{F}+\frac{1}{n} \log C_{e}
$$

Where, $Q_{e}(\mathrm{mg} / \mathrm{g})$ is amounts of dyes adsorbed at equilibrium, $C_{e}(\mathrm{mg} / \mathrm{L})$ is the concentration the dye at. $\mathrm{K}_{\mathrm{F}}$ and $(1 / \mathrm{n})$ are Freundlich isotherm constants. The Langmuir model, it is assumed that the dye undergoes monolayer adsorption around the homogenous surface of the adsorbent and that there is no interaction between the dyes molecules and the adsorbent. The Langmuir isotherm parameters were calculated using the following equation:

$$
\frac{C_{e}}{Q_{e}}=\frac{1}{Q_{\max } K_{L}}+\frac{C_{e}}{Q_{\max }}
$$

Where $C_{\mathrm{e}}\left(\mathrm{mg} \mathrm{L}^{-1}\right)$ is the equilibrium concentration of $\mathrm{CR}$ and FL dyes in solution, $Q_{\mathrm{e}}\left(\mathrm{mg} \mathrm{g}^{-1}\right)$ is the equilibrium adsorption capacity of CR and FL dyes on the FCS hydrogel, $Q_{\max }\left(\mathrm{mg} \mathrm{g}^{-1}\right)$ is the maximum amount of dyes on the FCS hydrogel to form monolayer on the surface, $K_{\mathrm{L}}\left(\mathrm{L} \mathrm{mg}^{-1}\right)$ is the Langmuir constant that relates to the affinity of adsorption active sites. 


\section{Results And Discussion}

\section{Characterization of FCS hydrogel}

The morphology of the FCS hydrogel of the fracture surface was analyzed by SEM, and the results were shown in Fig. 2. In the cross-sectional images of the FD-CS hydrogel, there are a highly porous matrix with interconnected pores extending from the core to the surface of the monolithic hydrogel, indicating the successful crosslinking of chitosan polymer chains to form chitosan composite hydrogels.

Furthermore, the prepared chitosan hydrogel with freeze-thawing treatment exhibited open pores with high interconnectivity structure. In contrast with other study which presented chitosan hydrogel with rough surface but with no pores were observed on the surface [25]. This is due to the freeze-thawing method has been used to create porous chitosan hydrogel with interconnected pores [42]. During cooling the prepared chitosan hydrogel, the nucleation and the ice crystals was grown and moves into nonnucleated solution leading to change the morphology and form interconnected pores [46]. At a temperature below the freezing temperature (room temperature), these ice crystals melt and gives rise to interconnected macroporous networks $[47,48]$. This network structure can improve the specific surface area and then increase the adsorption capacity of the dye molecules from aqueous media due to promoted diffusion of the dyes molecules within the hydrogel structure and makes contact between the functional groups of the chitosan hydrogel with dye molecules more efficient [49]. The specific surface area and pore size distributions of FDCS hydrogel was determined by employing the nitrogen sorption technique. The $\mathrm{N}_{2}$ adsorption-desorption isotherms of FDCS are represented in Fig. 3A.

The $\mathrm{N}_{2}$ adsorption-desorption isotherm for FCS belongs to type $\mathrm{H} 3$ hysteresis loop according to IUPAC classification, which proves interconnected pore structure with a parallel plate shape [50]. The synthesized FCS in this work displayed a specific surface area of $78.25 \pm 8.75 \mathrm{~m}^{2} \mathrm{~g}^{-1}$. The BET surface area of the prepared FCS chitosan compared with previous studies in the literature and it was found that the surface area for the FCS hydrogel was larger value [50-52]. These results strongly support the fact that the surface area and porosity of FCS were increased after freezing the CS hydrogel. Obviously, cryogenic treatment for the synthetic glutaraldehyde cross-linked chitosan hydrogel played an impressive role in the increasing of the surface area of this hydrogel. As expected, after freezing the CS hydrogel convert the porous structure from close pores to interconnected pores, which can allow more active sites in the hydrogel to interact with dye molecules and led to improve adsorption efficiency [53,54]. Therefore, FCS hydrogel with larger specific surface area and more active groups are a promising adsorbent materials due to these characteristics are particularly important for adsorption purposes for wastewater treatment [55]. The BET results well proved the SEM images as the pores development can be seen obviously in the surface morphology of FCS hydrogel. For a better evaluation of the impact of the initial CS concentrations on the porosity \% of FCS hydrogel, four different CS concentrations were used by fixing the crosslinking agent concentration constant. Increasing the CS concentration decreased the porosity of final FCS hydrogel as shown in Fig. 3B. The average porosity of FCS composite hydrogels decreases from $93.49 \%$ to $80.05 \%$ when the initial concentration of CS increases from 0.5 to $2.5 \%$ 
$\mathrm{w} / \mathrm{v}$. This is due to the fact that increasing the concentration of the polymer increases the cross-link densities of the network structure of the prepared hydrogel, which is lead to decrease the porosity [56].

In order to further confirm the successful crosslinking and the structural changes of composite hydrogel after crosslinking, the infrared spectra were investigated for powder chitosan and cross-linked chitosan hydrogel as shown in Fig. 3C. Comparison of the FTIR results reveals that the FCS cross-linked chitosan hydrogel shows spectra that are different from those of non-cross-linked chitosan. Non-cross-linked chitosan demonstrate

peaks at 949 and $1104 \mathrm{~cm}^{-1}$ are attributed to $\mathrm{C}-\mathrm{O}-\mathrm{C}$ bridge of chitosan unit. The absorption bands at 2911 and $2873 \mathrm{~cm}^{-1}$ can be corresponds to $\mathrm{C}-\mathrm{H}$ symmetric and asymmetric stretching. A broad band in the region of $3240-3400 \mathrm{~cm}^{-1}$ attributed to the associated of the vibrations of $-\mathrm{OH}$ and $-\mathrm{NH}_{2}$ groups. Band at about $1564 \mathrm{~cm}^{-1}$ are attributed to the bending of the primary amine's $\mathrm{N}-\mathrm{H}$ bond [57]. After chemical crosslinking of chitosan using glutaraldehyde, these band present a change in it is intensity and width, this is occurring due to the bond generated by overlapped the $\mathrm{N}-\mathrm{H}, \mathrm{O}-\mathrm{H}$, and $\mathrm{C}-\mathrm{O}$ bonds of $\mathrm{CS}$ with the GA during the crosslinking reaction. This proven that some of the hydrogen bonds in the chitosan structure are broken and new hydrogen bonds between GA and chitosan polymer chains are created as $\mathrm{NH}_{2}$ and $\mathrm{OH}$ functional groups engaged in hydrogen bonds [58]. A new peak at about $1646 \mathrm{~cm}^{-1}$ was observed, which is most probably attributed to the imine bond $(\mathrm{C}=\mathrm{N})$. This bond produces and observed in the spectrum of FCS hydrogel due the chemical crosslinking reaction between GA and CS amino groups [26]. The FTIR results confirms that GA cross-linking takes place by the reaction of amine groups of chitosan with aldehyde groups of glutaraldehyde. Fig. S1 shows change of the chitosan hydrogel color from transparent light yellow to orange this was also confirms the formation of cross-links between CS polymer chains by GA. This is most likely due to the formation of a chromophore as stable crosslinking products, which is not present in the starting precursors [59]. The Z-potential of FCS hydrogel in deionized water was measured by the DLS of suspensions that were prepared by dispersing $0.05 \mathrm{~g}$ of the vacuum dried FCS hydrogel in $50 \mathrm{~mL}$ of deionized water by a digital sonicator. The zeta potential of FCS hydrogel was found to be $+26.5 \pm 3 \mathrm{mV}$ as shown in Fig. 3D. At this point, the positive surface charge of FCS hydrogel contributed to increase the adsorption capacity towards the anionic contaminated and this result agrees with previous studies [60-62].

It is important to highlight that the positive value of ZP for FCS hydrogel indicating that the high protonation of the $\mathrm{NH}_{2}$ groups, which are did not take part in the crosslinking reaction and suggests that the interactions between the FCS hydrogel and anionic dyes molecules may occur by hydrogen bonding and/or electrostatic interactions [63].

The swelling behavior of the FCS hydrogel was analyzed via gravimetric method. In detail, a small piece of vacuum dried hydrogel $(\sim 0.05 \mathrm{~g})$ was immersing in D.I. water for 24 hours at room temperature $(25 \pm 3$ ${ }^{\circ} \mathrm{C}$ ) and neutral $\mathrm{pH}$. The FCS hydrogel shows remarkable and quick volume change due to the water pass through the hydrogel via capillary forces. This was owing to its interconnected porous structure and the presence of abundance hydrophilic functional groups on backbone [64]. Swelling behavior of FCS 
hydrogels as a function of $\mathrm{pH}$ was evaluated as shown in Fig. 4. As can be seen in Fig. 4A the swelling ratio of FCS hydrogels with decreasing the $\mathrm{pH}$ value from 12 to 3 was determined. The swelling ratio of FCS hydrogels in $\mathrm{pH} 3$ was 26.3, which doubled compared to the same sample in $\mathrm{pH} 12$ (11.7). It is worth noting that, FCS hydrogel in acidic environment showed higher swelling ratio than the same sample in basic and neutral environment. In order to explain this phenomenon, it was due to in acidic media ionized the $-\mathrm{NH}_{2}$ groups in the FCS hydrogel to ammonium ion $\left(\mathrm{NH}_{3}{ }^{+}\right)$and these cationic charges made repulsive forces between polymer chains [65]. Fig. 4B shows the swelling deswelling reversibility of FCS hydrogel between $\mathrm{pH} 3$ and $\mathrm{pH} 10$. The results demonstrated that the reversibility toward the $\mathrm{pH}$ changes was retained even after more than ten cycles. FCS hydrogel water uptake were studied by measuring the degree of swelling with time. Fig. $4 \mathrm{C}$ depicts the degree of swelling as a function of time for FCS hydrogels in acidic media. Fig. 4D shows deswelling kinetics of the FCS hydrogel at pH 10. FCS hydrogels exhibited quicker loss most of water after change the $\mathrm{pH}$ value from 3 to 10 in comparison with water uptake of the same FCS sample. This results are reasonable since both swelling and deswelling processes start from the surface of the hydrogels to inside the hydrogel through its porous structure. During swelling, water will take longer time to penetrate inside the sample, while it will take a shorter time for the transportation from the hydrogel during deswelling. Fig. 4E demonstrated schematic represented the swelling and deswelling properties of FCS hydrogel in acidic and alkaline conditions respectively.

\section{Adsorption study}

The synthesized three-dimensional FCS hydrogel with unique pore structure were evaluated for the removal of some organic dyes. In the present work, Congo Red and Sodium Fluorescent dyes as an anionic dye model and Methylene Blue and Rodamine $6 \mathrm{G}$ as cationic dye model to test the ability of FCS hydrogel to remove organic dyes. It is also important to note that the preparation process of our FCS hydrogel requires only mixing of chitosan and the chemical cross linker in acidic media and then freezethaw treatment to generate the pores, with no further drying step required to form the hydrogel absorbent. This low energy fabrication method, together with the use of the green solvent, water, renders our approach relatively environmentally friendly. Further, we described the dye adsorption process utilizes monolithic hydrogel, with no required for the additional steps of centrifugation or adsorbent separation, unlike methods that utilized particulate-based absorbents. Fig. S2 show a photograph of the adsorption process of CR and SFL by FCS monolithic hydrogels. After immersing the FCS monolithic hydrogel by 24 hours into CR and SFL dyes solutions separately, the red and yellow solutions containing CR and SFL dyes become very light and almost clear and transparent, whereas the hydrogels turned red and dark yellow due to the adsorption of CR and SFL dyes respectively. These images indicate that the composite FCS pores hydrogels possess highly efficient of anionic dyes adsorption. In contrast, FCS hydrogel demonstrates very low removal towards MB and R6G as shown in Fig. S3. However, the monolithic FCS hydrogel turned to blue and dark orange after immersing these hydrogels into MB and R6G solutions by 24 hours. The reason for this is due to the interconnected porous structure of FCS hydrogel make easily filled by these dyes solutions without being adsorbed [66]. The adsorption capacities of the FCS hydrogel toward CR and SFL dyes were evaluated by using the UV-Vis spectra to measure the concentrations of 
the dye solution before and after adsorption as shown in Fig. $5 \mathrm{~A}$ and B. Removal and adsorption of CR and SFL dyes onto the FCS monolithic hydrogels from the aqueous solution was observed. UV-Vis spectroscopy shows low absorption bands for these two dyes in the solution after adsorption. This phenomenon may be explained by electrostatic attraction between $\mathrm{NH}_{3}{ }^{+}$group protonated in the acid medium of FCS hydrogel and anionic dye molecules formation $\left(\right.$ Dye- $\left.\mathrm{NH}_{3}{ }^{+}\right)$, which is cause the captured of this type of dyes on FCS hydrogel [3]. We discovered that the hydrogen bonding and electrostatic interactions played a significant role in the adsorption of the anionic dyes [67]. Similarly, FCS hydrogel adsorption capacity were tested towards $\mathrm{MB}$ and R6G dyes aqueous solutions as cationic dyes. In contrast to CR and SFL dyes, no significant adsorption was observed toward MB and R6G (less than $10 \%$ ), with little change in the UV-Vis spectrum of the dyes solutions after adsorption as shown in Fig. $5 \mathrm{C}$ and D.

This confirmed that FCS monolithic hydrogel has no obvious adsorption of cationic dyes on its surface due to the electrostatic repulsion interactions between the dyes and the cationic FCS hydrogel [67]. However, it should be noted that the poor removal efficiency of cationic dye due to the hydroxyl groups in the FCS may also participate to chelation with cationic dyes [68]. In order to study the adsorption behavior of the FCS porous monolithic hydrogel towards CR and SFL dyes batch adsorption method was also performed. The batch adsorption of these two anionic dyes were determined at different initial concentrations with constant weight of FCS hydrogel $(0.05 \mathrm{~g})$ for different adsorption times intervals at a pH value of 6 . As shown in Fig. 6A, demonstrates the adsorption process of both $\mathrm{CR}$ and SFL dyes (the concentrations were $60 \mathrm{mg} / \mathrm{L}$ ) onto the FCS monolithic hydrogel were progressed rapidly in the initial stage and the adsorption rate decreased gradually moving toward equilibrium after 12 hours. The best possible explanation for this phenomenon is that at the beginning of the reaction, the higher available active site with the higher driving force on the FCS hydrogels surfaces provides better opportunity of collision between anionic dyes molecules and the adsorbent [69]. As the adsorption reaction reached equilibrium the adsorption capacity was remained constant, which is may be attributed to saturated the available adsorption sites of the FCS hydrogel by dyes molecules [70]. The adsorption capacity at equilibrium of CR and SFL dyes were calculated and it were found 104.9 and $99.6 \mathrm{mg} . \mathrm{g} \mathrm{g}^{-1}$ respectively. The removal percentage of CR and SFL dyes were up to $96.73 \%$ and $95 \%$ at equilibrium as shown in Fig. 6B. The study of the adsorption isotherm play a significant role to describe how adsorbates molecules interact with adsorbents surface [71]. In the present work, to investigate the equilibrium condition of anionic dyes (CR and SFL) onto FCS hydrogel, two types of isotherms including Freundlich and Langmuir isotherm models were evaluated. The Langmuir isotherm model can be regarded as a single layer adsorption system onto a homogeneous surface, while the Freundlich model are considered as a heterogeneous multi layers adsorption system [72]. As we can see in Fig. $6 \mathrm{C}$ and D the plots of Langmuir and Freundlich isotherm for CR and SFL dyes. The fitted parameters and data are shown in Table S1. The results indicate that the Freundlich adsorption isotherm showed a better value of correlation coefficient $\left(\mathrm{R}^{2}\right)$. Therefore, the adsorption of both CR and SFL dyes onto FCS hydrogel obeyed with the Freundlich model, and is mainly multilayer adsorption. Through the Langmuir model fitting, the maximum adsorption capacity $\mathrm{q}_{\mathrm{m}}$ values for $\mathrm{CR}$ and $\mathrm{SFL}$ dyes onto FCS hydrogel were obtained and it 
were found 136.69 and $142.857 \mathrm{mg}^{-1} \mathrm{~g}^{-1}$ respectively. The small difference in maximum adsorption capacities of these dyes was owing to their different affinities for adsorbance.

As a results, the high adsorption capacity of FCS hydrogel due to the adsorption mechanism is affected by the interconnected porous structure of the hydrogel, which is allow better access of dye molecules through the internal structure of the hydrogel and increase the available active adsorption sites [73].

Additionally, the strong electrostatic force between the positively charged amine groups $\left(-\mathrm{NH}_{3}{ }^{+}\right)$in the hydrogel FCS interact with the negatively charged groups on anionic dyes and the like hydrogen bonds interaction encourage anionic dyes adsorption on hydrogels resulting high adsorption capacity $[16$, 74]. To preform, the adsorption behavior of the FCS hydrogel, kinetic models containing pseudo-first order (PFO) and pseudo-second-order (PSO) models, which are widely used in the fitting of experimental kinetics data as shown in Table S2 and S3 for CR and SFL dyes respectively. The fitting plots of the adsorption kinetic model is shown in Fig. 7. It was observed that the correlation coefficient $\left(R^{2}\right)$ corresponding to the PSO model was significantly higher than that corresponding to the PFO model. Furthermore, the results showed that the simulation adsorption capacity from the plot of PSO model was found to be well fit with the experimental results than that calculated from PFO. Therefore, PSO model better describe the adsorption mechanism for both CR and SFL dyes on FCS hydrogel. It is worth to note that the adsorption reaction of CR and SFL dyes on to FCS hydrogel is more tending towards physical adsorption. Basically, the adsorption mechanism of dye molecules on a porous hydrogel adsorbent material cannot be described by only the PFO and PSO models due to the adsorption on these materials may include a multi-stage process [75]. As a result, an intraparticle diffusion model was employed for further interpretation to show the movement of the dye molecules from the aqueous solution into the interconnected pores of the FCS hydrogel. Fig. 7E and F show that the linear plote do not pass through the origin for both CR and SFL dyes, indicating that the intraparticle diffusion models is based on the migration of the adsorbates into the hydrogel pores and controlling the rate adsorption process and the results of adsorbent parameters for intraparticle diffusion model were listed Table S2 and S3 for CR and SFL dyes respectively. This model represents two stages, the first stage where there is a rapid adsorption of dye molecules on the external surface of the hydrogel and the second stage where the intraparticle diffusion is the rate-limiting step [76]. Therefore, in addition to PSO, the intraparticle diffusion model plays a significant role to interpret the adsorption mechanism.

\section{Reusability and desorption studies}

In order to evaluate the reusability and regeneration of FCS monolithic hydrogel as adsorbents to be a cost effective material, desorption experiments were carried out using FCS hydrogel as adsorbent and CR and SFL dyes as adsorbates. So the desorption of adsorbed CR and SFL dyes from FCS monolithic hydrogel was investigated in a batch desorption system. Because the FCS is a monolithic hydrogel, the collection of the adsorbent was fast and easy. The FCS hydrogel was recovered by immersing in a $0.01 \mathrm{M}$ $\mathrm{NaOH}$ solution for $6 \mathrm{~h}$ to desorb CR and SFL dyes that had adsorbed on the hydrogel. Then the hydrogel was protonated in excess $0.01 \mathrm{M} \mathrm{HCl}$ for $1 \mathrm{~h}$ to regenerate cationic active sites to use in the next 
adsorption cycle. The regenerated FCS was further washed by deionization water to reach neutrality. The reusability of FCS hydrogel was evaluated over five cycles of adsorption/desorption by using $\mathrm{NaOH}$ solution as the desorption agents with $0.05 \mathrm{~g} \mathrm{FCS} \mathrm{monolithic} \mathrm{hydrogel} \mathrm{in} 100 \mathrm{~mL}$ dye solution (10 ppm) at $25^{\circ} \mathrm{C}$ for $24 \mathrm{~h}$ (each cycle), and the results are shown in Fig. 8. As seen, no significant losses in the dye adsorption performance was detected even after five cycles of reusability. It was observed that about $9 \%$ and $3 \%$ reduction in removal efficiency of FCS hydrogel for CR and SFL dyes respectively.

Thus, the results depict that the electrostatic attraction is dominating driving force in the adsorption process in this study. It is important to highlight that the positively charged amino group in FCS hydrogel was deprotonated in alkaline conditions, which is affecting the electrostatic interactions between FCS hydrogel and anionic dye and then desorbed the loaded dye from adsorption active site [77]. Therefore, it can be concluded that FCS monolithic hydrogel promising ecofriendly adsorbent material due to can be regenerated easily and used repeatedly as an efficient adsorbent for water purification.

\section{Conclusion}

In the present study, macroporous FCS hydrogel was successfully prepared using a new and simple freeze-thaw strategy as physical modification of glutaraldehyde cross-linked chitosan hydrogel with the aim of fabrication interconnected porous hydrogel. This porous structure in the hydrogel materials improves the accessibility of adsorbates in the hydrogel active sites and increases the dye adsorption capacity of the FCS hydrogel. To the best of our knowledge, there are no studies regarding the use of the freeze-thaw method for the fabrication of macroporous chitosan hydrogel with interconnected pores. The FCS hydrogel was characterized and the results demonstrated that FCS hydrogel had a highly porous matrix with interconnected pores extending from the core to the surface of the monolithic hydrogel as revealed by SEM. The swelling-deswelling behavior of FCS hydrogel was investigated and the results indicated that the FCS hydrogel exhibited good swelling properties in acidic media than the same sample in basic media. The reason behind that the amine groups $\left(-\mathrm{NH}_{2}\right)$ of chitosan in acidic medium protonation to ammonium ion $\left(\mathrm{NH}_{3}{ }^{+}\right)$due to the presence of hydrogen ions and these cationic charges made repulsive forces between polymer chains and increase the capillary force. The swelling deswelling reversibility results of FCS hydrogel showed a significant $\mathrm{pH}$ dependent, which demonstrated that the reversibility toward the $\mathrm{pH}$ changes was retained even after more than ten cycles. The detailed evaluation of adsorption behavior of different organic dyes by FCS hydrogel revealed that this hydrogel possesses the unique adsorption toward anionic dyes with a large adsorption capacity, but very low adsorption capacity toward cationic dyes. The electrostatic interactions dominated the interaction between the FCS hydrogel and the dye molecules regardless to their charge states. The adsorption isotherms data of $\mathrm{CR}$ and SFL onto FCS showed a better fit with the Ferunditich model. The adsorption kinetics analysis exhibited that the adsorption process could be described well with the pseudo-second-order model. The obtained FCS provided not only selective absorption towards anionic dyes with a high removal efficiency but also exhibited excellent adsorption efficiency with no significant loss even after five cycles of reusability. From the obtained results, the synthesized FCS hydrogel by the freeze-thaw method is 
considered to be a promising candidate for the removal of anionic dyes from an aqueous solution due to its high adsorption capacity and cost-effective properties.

\section{Declarations}

\section{Notes}

The authors declare no competing financial interest.

\section{Acknowledgments}

The authors would like to thank the University of Babylon, College of Science in Iraq for funding this work as a part of an annual research plan for academic staff. Z.H.A. and A.F.H. would like to express their acknowledgment to the University of Hull.

\section{References}

1. Zhao J, Yin J, Zhong J, et al (2019) Facile preparation of a self-assembled artemia cyst shell- $\mathrm{TiO}_{2}-$ $\mathrm{MoS}_{2}$ porous composite structure with highly efficient catalytic reduction of nitro compounds for wastewater treatment. Nanotechnology 31:85603

2. Wang C, Yin J, Han S, et al (2019) Preparation of palladium nanoparticles decorated polyethyleneimine/polycaprolactone composite fibers constructed by electrospinning with highly efficient and recyclable catalytic performances. Catalysts 9:559

3. Binaeian E, Zadvarzi SB, Yuan D (2020) Anionic dye uptake via composite using chitosanpolyacrylamide hydrogel as matrix containing $\mathrm{TiO}_{2}$ nanoparticles; comprehensive adsorption studies. Int J Biol Macromol 162:150-162

4. Islam MA (2020) Synthesis of manganite $(\mathrm{\gamma}-\mathrm{MnOOH})$ for the adsorptive degradation of methylene blue (MB) and orange green (OG) from aqueous solution. Cell 1818:482533

5. Kapoor RT, Danish M, Singh RS, et al (2021) Exploiting microbial biomass in treating azo dyes contaminated wastewater: Mechanism of degradation and factors affecting microbial efficiency. $J$ Water Process Eng 43:102255

6. Demirbas A (2009) Agricultural based activated carbons for the removal of dyes from aqueous solutions: A review. J Hazard Mater 167:1-9. https://doi.org/https://doi.org/10.1016/j.jhazmat.2008.12.114

7. Gupta VK, Suhas (2009) Application of low-cost adsorbents for dye removal - A review. J Environ Manage 90:2313-2342. https://doi.org/https://doi.org/10.1016/j.jenvman.2008.11.017

8. Vashi H, lorhemen OT, Tay JH (2017) Aerobic granulation: A recent development on the biological treatment of pulp and paper wastewater. Environ Technol Innov 9:265-274

9. An B, Choi J (2019) An Experimental Application of Four Types of Chitosan Bead for Removal of Cationic and Anionic Pollutants. Air and Soil Pollution 230(12): 1-13 
10. Mezohegyi G, van der Zee FP, Font J, et al (2012) Towards advanced aqueous dye removal processes: a short review on the versatile role of activated carbon. J Environ Manage 102:148-164. https://doi.org/10.1016/j.jenvman.2012.02.021

11. Halbus AF, Athab ZH, Hussein FH (2021) Egyptian Journal of Chemistry. 64:7255-7268. https://doi.org/10.21608/ejchem.2021.79055.3870

12. Hussein FH, Halbus AF, Lafta AJ, Athab ZH (2015) Preparation and characterization of activated carbon from iraqi khestawy date palm. J Chem 2015:. https://doi.org/10.1155/2015/295748

13. Zahraa H.A. (2015) Production and Characterization of Activated Carbon From Iraqi Palm Fiber. Asian J Chem 27:3658-3662

14. Halbus AF, Athab ZH, Hussein FH (2013) Adsorption of disperse blue dye on Iraqi date palm seeds activated carbon. Int J Chem Sci 11:1219-1233

15. Ren J, Wang X, Zhao L, et al (2021) Effective Removal of Dyes from Aqueous Solutions by a Gelatin Hydrogel. J Polym Environ 1-12

16. Sinha V, Chakma S (2019) Advances in the preparation of hydrogel for wastewater treatment: A concise review. J Environ Chem Eng 7:103295

17. Hou N, Wang R, Geng R, et al (2019) Facile preparation of self-assembled hydrogels constructed from poly-cyclodextrin and poly-adamantane as highly selective adsorbents for wastewater treatment. Soft Matter 15:6097-6106

18. Loo S-L, Vásquez L, Athanassiou A, Fragouli D (2021) Polymeric Hydrogels-A Promising Platform in Enhancing Water Security for a Sustainable Future. Adv Mater Interfaces n/a:2100580. https://doi.org/https://doi.org/10.1002/admi.202100580

19. Weerasundara L, Gabriele B, Figoli A, et al (2020) Hydrogels: Novel materials for contaminant removal in water-A review. Crit Rev Environ Sci Technol 51:1-45. https://doi.org/10.1080/10643389.2020.1776055

20. Kaith BS, Singh A, Sharma AK, Sud D (2021) Hydrogels: Synthesis, Classification, Properties and Potential Applications-A Brief Review. J Polym Environ 1-15

21. Yu Y, Cheng Y, Tong J, et al (2021) Recent advances in thermo-sensitive hydrogels for drug delivery. J Mater Chem B 9:2979-2992

22. Qi X, Tong X, Pan W, et al (2021) Recent advances in polysaccharide-based adsorbents for wastewater treatment. J Clean Prod 315:128221. https://doi.org/https://doi.org/10.1016/j.jclepro.2021.128221

23. Hassanzadeh P, Gharbani P, Derakhshanfard F, Maher BM (2021) Preparation and Characterization of PVDF/gC 3 N 4/Chitosan Polymeric Membrane for the Removal of Direct Blue 14 Dye. J Polym Environ 1-10

24. Younes I, Rinaudo M (2015) Chitin and chitosan preparation from marine sources. Structure, properties and applications. Mar Drugs 13:1133-1174 
25. Ostrowska-Czubenko J, Gierszewska M, Pieróg M (2015) pH-responsive hydrogel membranes based on modified chitosan: water transport and kinetics of swelling. J Polym Res 22:1-12

26. Galan J, Trilleras J, Zapata PA, et al (2021) Optimization of Chitosan Glutaraldehyde-Crosslinked Beads for Reactive Blue 4 Anionic Dye Removal Using a Surface Response Methodology. Life 11

27. Chang X, Chen D, Jiao X (2008) Chitosan-based aerogels with high adsorption performance. J Phys Chem B 112:7721-7725

28. Bratskaya S, Privar Y, Nesterov D, et al (2019) Chitosan Gels and Cryogels Cross-Linked with Diglycidyl Ethers of Ethylene Glycol and Polyethylene Glycol in Acidic Media. Biomacromolecules 20:1635-1643. https://doi.org/10.1021/acs.biomac.8b01817

29. Yang C, Shi X, Qi L, et al (2021) Electrical Writing Induced Covalent Cross-Linking on Hydrogel for Multidimensional Structural Information Storage. ACS Appl Mater Interfaces 13:36538-36547. https://doi.org/10.1021/acsami.1c09548

30. Pérez-Calderón J, Santos MV, Zaritzky N (2020) Synthesis, characterization and application of crosslinked chitosan/oxalic acid hydrogels to improve azo dye (Reactive Red 195) adsorption. React Funct Polym 155:104699

31. Mathew AP, Laborie M-PG, Oksman K (2009) Cross-Linked Chitosan/Chitin Crystal Nanocomposites with Improved Permeation Selectivity and pH Stability. Biomacromolecules 10:1627-1632. https://doi.org/10.1021/bm9002199

32. Kayan GÖ, Kayan A (2021) Composite of Natural Polymers and Their Adsorbent Properties on the Dyes and Heavy Metal lons. J Polym Environ 1-20

33. Reghioua A, Barkat D, Jawad AH, et al (2021) Magnetic Chitosan-Glutaraldehyde/Zinc Oxide/Fe 30 4 Nanocomposite: Optimization and Adsorptive Mechanism of Remazol Brilliant Blue R Dye Removal. J Polym Environ 1-16

34. Tu H, Yu Y, Chen J, et al (2017) Highly cost-effective and high-strength hydrogels as dye adsorbents from natural polymers: chitosan and cellulose. Polym Chem 8:2913-2921

35. Mahmoodi H, Fattahi M, Motevassel M (2021) Graphene oxide-chitosan hydrogel for adsorptive removal of diclofenac from aqueous solution: preparation, characterization, kinetic and thermodynamic modelling. RSC Adv 11:36289-36304

36. Alves DCS, Goncalves JO, Coseglio BB, et al (2019) Adsorption of phenol onto chitosan hydrogel scaffold modified with carbon nanotubes. J Environ Chem Eng 7:103460

37. Alves DC, Healy B, Yu T, Breslin CB (2021) Graphene-based materials immobilized within Chitosan: Applications as adsorbents for the removal of aquatic pollutants. Materials (Basel) 14:3655

38. Niu Z, Liu L, Zhang L, Chen X (2014) Porous graphene materials for water remediation. Small 10:3434-3441

39. da Silva Alves DC, Healy B, Pinto LA, et al (2021) Recent developments in chitosan-based adsorbents for the removal of pollutants from aqueous environments. Molecules 26:594 
40. Chatterjee S, Chatterjee T, Lim S-R, Woo SH (2011) Effect of the addition mode of carbon nanotubes for the production of chitosan hydrogel core-shell beads on adsorption of Congo red from aqueous solution. Bioresour Technol 102:4402-4409

41. Annabi N, Nichol JW, Zhong X, et al (2010) Controlling the porosity and microarchitecture of hydrogels for tissue engineering. Tissue Eng Part B Rev 16:371-383. https://doi.org/10.1089/ten.TEB.2009.0639

42. Xue C, Wilson LD (2021) An Overview of the Design of Chitosan-Based Fiber Composite Materials. J Compos Sci 5:160

43. Sun S, Tang Y, Fu Q, et al (2012) Preparation of agarose/chitosan composite supermacroporous monolithic cryogels for affinity purification of glycoproteins. J Sep Sci 35:893-900

44. Ho M-H, Kuo P-Y, Hsieh H-J, et al (2004) Preparation of porous scaffolds by using freeze-extraction and freeze-gelation methods. Biomaterials 25:129-138

45. Quirk RA, France RM, Shakesheff KM, Howdle SM (2004) Supercritical fluid technologies and tissue engineering scaffolds. Curr Opin Solid State Mater Sci 8:313-321

46. Deville S, Saiz E, Nalla RK, Tomsia AP (2006) Freezing as a path to build complex composites. Science (80- ) 311:515-518

47. Sornkamnerd S, Okajima MK, Kaneko T (2017) Tough and porous hydrogels prepared by simple lyophilization of LC gels. ACS omega 2:5304-5314

48. Hwang Y, Sangaj N, Varghese S (2010) Interconnected macroporous poly (ethylene glycol) cryogels as a cell scaffold for cartilage tissue engineering. Tissue Eng Part A 16:3033-3041

49. Ding Y, Song C, Gong W, et al (2021) Robust, sustainable, hierarchical multi-porous cellulose beads via pre-crosslinking strategy for efficient dye adsorption. Cellulose 1-15

50. Luo W, Bai Z, Zhu Y (2018) Fast removal of Co (ii) from aqueous solution using porous carboxymethyl chitosan beads and its adsorption mechanism. RSC Adv 8:13370-13387

51. Azlan K, Saime WNW, Liew LAl (2009) Chitosan and chemically modified chitosan beads for acid dyes sorption. J Environ Sci 21:296-302

52. Pivarčiová L, Rosskopfová O, Galamboš M, et al (2016) Sorption of pertechnetate anions on chitosan. J Radioanal Nucl Chem 308:93-98

53. Dui J, Zhu G, Zhou S (2013) Facile and economical synthesis of large hollow ferrites and their applications in adsorption for As (V) and Cr (VI). ACS Appl Mater Interfaces 5:10081-10089

54. Zhang Y, Yan W, Sun Z, et al (2015) Fabrication of porous zeolite/chitosan monoliths and their applications for drug release and metal ions adsorption. Carbohydr Polym 117:657-665

55. Song W, Xu J, Gao L, et al (2021) Preparation of Freeze-Dried Porous Chitosan Microspheres for the Removal of Hexavalent Chromium. Appl Sci 11:4217

56. Kizilay MY, Okay O (2003) Effect of initial monomer concentration on spatial inhomogeneity in poly (acrylamide) gels. Macromolecules 36:6856-6862 
57. Nematidil N, Sadeghi M, Nezami S, Sadeghi H (2019) Synthesis and characterization of Schiff-base based chitosan-g-glutaraldehyde/NaMMTNPs-APTES for removal $\mathrm{Pb}^{2+}$ and $\mathrm{Hg}^{2+}$ ions. Carbohydr Polym 222:114971

58. Ostrowska-Czubenko J, Gierszewska-Drużyńska M (2009) Effect of ionic crosslinking on the water state in hydrogel chitosan membranes. Carbohydr Polym 77:590-598

59. Roberts GAF, Taylor KE (1989) Chitosan gels, 3. The formation of gels by reaction of chitosan with glutaraldehyde. Die Makromol Chemie Macromol Chem Phys 190:951-960

60. Raju L, AR SCL, Prakash NKU, Rajkumar E (2021) Chitosan-terephthaldehyde hydrogels-Effect of concentration of cross-linker on structural, swelling, thermal and antimicrobial properties. Materialia 16:101082

61. Leduc J-F, Leduc R, Cabana H (2014) Phosphate adsorption onto chitosan-based hydrogel microspheres. Adsorpt Sci Technol 32:557-569

62. Saïed N, Aïder M (2014) Zeta potential and turbidimetry analyzes for the evaluation of chitosan/phytic acid complex formation. $J$ food Res 3:71

63. Boardman SJ, Lad R, Green DC, Thornton PD (2017) Chitosan hydrogels for targeted dye and protein adsorption. J Appl Polym Sci 134:

64. Lone S, Yoon DH, Lee H, Cheong IW (2019) Gelatin-chitosan hydrogel particles for efficient removal of $\mathrm{Hg}$ (II) from wastewater. Environ Sci Water Res Technol 5:83-90

65. Park H, Park K, Kim D (2006) Preparation and swelling behavior of chitosan-based superporous hydrogels for gastric retention application. J Biomed Mater Res Part A An Off J Soc Biomater Japanese Soc Biomater Aust Soc Biomater Korean Soc Biomater 76:144-150

66. Ullah F, Othman MBH, Javed F, et al (2015) Classification, processing and application of hydrogels: A review. Mater Sci Eng C 57:414-433. https://doi.org/https://doi.org/10.1016/j.msec.2015.07.053

67. Wu M, Chen W, Mao Q, et al (2019) Facile synthesis of chitosan/gelatin filled with graphene bead adsorbent for orange II removal. Chem Eng Res Des 144:35-46

68. Domard A (1987) Determination of $\mathrm{N}$-acetyl content in chitosan samples by cd measurements. Int J Biol Macromol 9:333-336

69. Hameed BH, El-Khaiary MI (2008) Malachite green adsorption by rattan sawdust: Isotherm, kinetic and mechanism modeling. J Hazard Mater 159:574-579

70. Zhao R, Zheng H, Zhong Z, et al (2021) Efficient removal of diclofenac from surface water by the functionalized multilayer magnetic adsorbent: Kinetics and mechanism. Sci Total Environ 760:144307. https://doi.org/https://doi.org/10.1016/j.scitotenv.2020.144307

71. Hosseinzadeh H, Ramin S (2018) Effective removal of copper from aqueous solutions by modified magnetic chitosan/graphene oxide nanocomposites. Int J Biol Macromol 113:859-868

72. Yang S, Zhao F, Sang Q, et al (2021) Investigation of 3-aminopropyltriethoxysilane modifying attapulgite for Congo red removal: Mechanisms and site energy distribution. Powder Technol 383:74-83. https://doi.org/https://doi.org/10.1016/j.powtec.2021.01.046 
73. Kıvanç MR, Ozay O, Ozay H, llgin P (2020) Removal of anionic dyes from aqueous media by using a novel high positively charged hydrogel with high capacity. J Dispers Sci Technol 1-16

74. Puchana-Rosero MJ, Adebayo MA, Lima EC, et al (2016) Microwave-assisted activated carbon obtained from the sludge of tannery-treatment effluent plant for removal of leather dyes. Colloids Surfaces A Physicochem Eng Asp 504:105-115

75. Pan M, Lin X, Xie J, Huang X (2017) Kinetic, equilibrium and thermodynamic studies for phosphate adsorption on aluminum hydroxide modified palygorskite nano-composites. RSC Adv 7:4492-4500

76. Zhou C, Wu Q, Lei T, Negulescu II (2014) Adsorption kinetic and equilibrium studies for methylene blue dye by partially hydrolyzed polyacrylamide/cellulose nanocrystal nanocomposite hydrogels. Chem Eng J 251:17-24

77. Chiou MS, Li HY (2003) Adsorption behavior of reactive dye in aqueous solution on chemical crosslinked chitosan beads. Chemosphere 50:1095-1105

\section{Figures}

\section{Figure 1}

Schematic illustration of $(A)$ the synthesis of cationic chitosan with interconnected hydrogel using glutaraldehyde as a crosslinking agent and treated by freezing and thawing method (B) its application for removal of anionic dye by adsorption (Congo Red dye as typical model for anionic dyes).

\section{Figure 2}

SEM cross-sectional images of the FCS hydrogel dried by freeze drying (A-D) at different magnifications and scales.

\section{Figure 3}

(A) $\mathrm{N}_{2}$ adsorption/desorption isotherms of FCS hydrogel (B) FTIR spectrum of powder chitosan and glutaraldehyde cross linked chitosan (C) Bar charts showing porosity of FCS hydrogel with different CS concentration (D) The zeta potential of FCS hydrogel was dispersed in deionized water at $\mathrm{pH} 7$. 


\section{Figure 4}

(A) Swelling degree (SD) as a function of $\mathrm{pH}$ of the FCS hydrogel at room temperature (B) Reversibility cycle of swelling-deswelling of FCS hydrogel as a function of time by varying the $\mathrm{pH}$ from 3 to 10 (C) Water uptake ratio (WU) for the FCS hydrogel as a function of time, when plunged in a $\mathrm{pH} 3$ after initial equilibration at $\mathrm{pH}$ 10. (D) Water retention ratio (WR) of FCS hydrogel as a function of time, when plunged in a pH 10 after initial equilibration at pH 3. (E) Schematic illustration of swelling and deswelling behavior of the FCS hydrogel in wet states at different $\mathrm{pH}$ and at $25^{\circ} \mathrm{C}$. Corresponding optical image of the shrunk and swollen states of FCS hydrogel in basic and acidic media.

\section{Figure 5}

Selective adsorption behaviors toward CR, SFL, MB and R6G dyes: UV-Vis spectra of CR (A), SFL (B), MB (C) and R6G (D) before and after adsorption by using FCS hydrogel for $24 \mathrm{~h}$. Insets are the images of CR, SFL, MB, and R6G dyes solutions before and after adsorption for 24 hours.

\section{Figure 6}

(A) The effect of adsorption time on adsorption capacity of CR and SFL by FCS hydrogels (the initial dye concentration: $60 \mathrm{mg} / \mathrm{L}$, adsorbent dosage: $0.05 \mathrm{~g}$ in $100 \mathrm{~mL}$ of dye solutions, $\mathrm{pH}$ of $6, \mathrm{t}=25^{\circ} \mathrm{C}$, shaking rate is constant at $100 \mathrm{rpm}$ ). (B) The effect of contact time on the removal percentage of CR and SFL dyes of dye (C) and (D) Freundlich and Langmuir adsorption isotherms respectively.

\section{Figure 7}

Kinetic models for adsorption of CR and SFL dyes using FCS hydrogel (A) and (B) pseudo-first order model for CR and SFL dyes respectively (C) and (D) pseudo-second order model for CR and SFL dyes respectively $(E)$ and $(F)$ intraparticle diffusion model for $C R$ and SFL dyes respectively. 


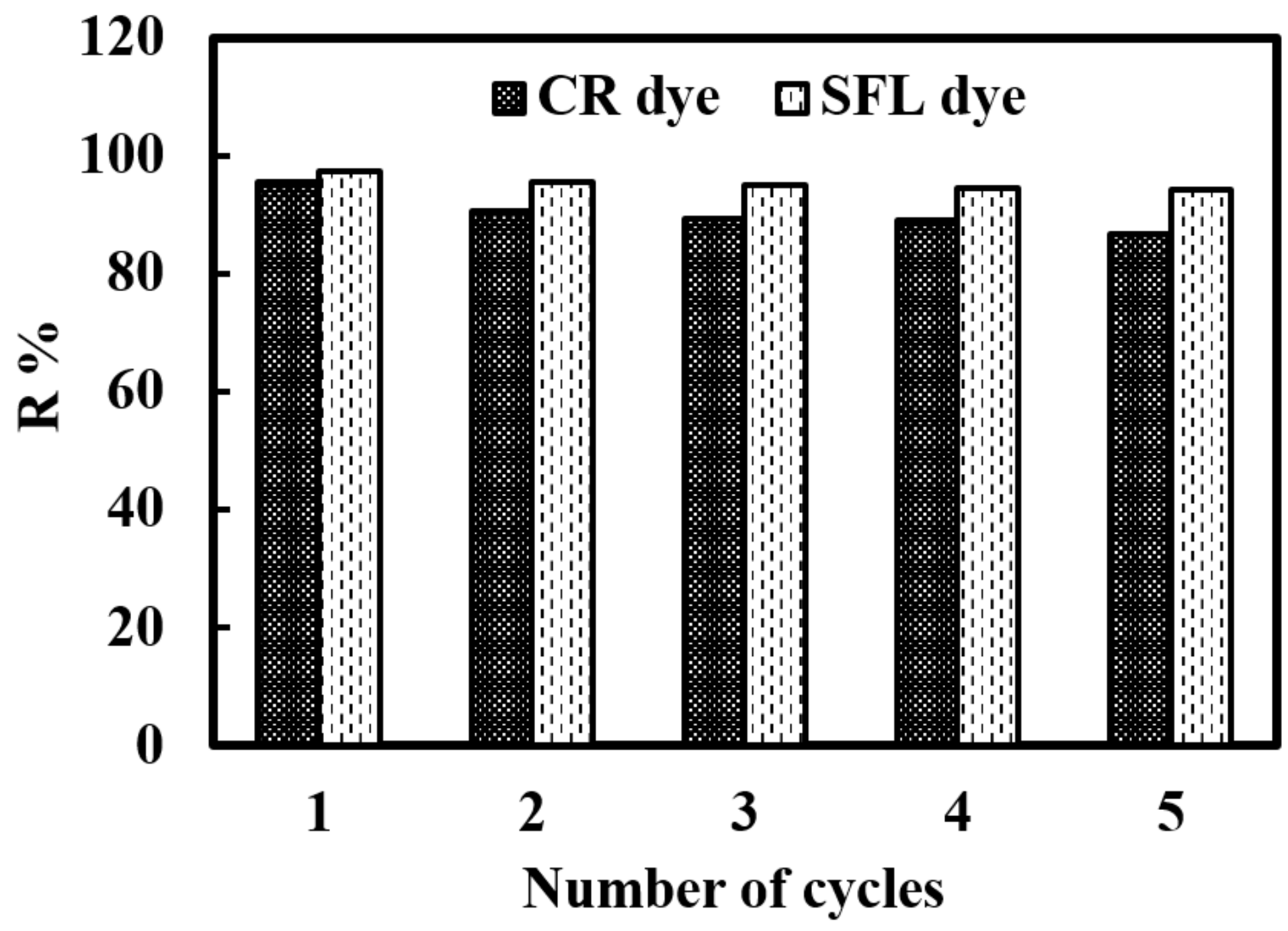

Figure 8

Reusability of FCS monolithic hydrogel for CR and SFL dyes (FCS dosage $=0.05 \mathrm{~g}$; dye concentration $=10$ $\mathrm{mg} / \mathrm{Lin} 100 \mathrm{~mL} ; \mathrm{pH}=6 ; \mathrm{t}=25^{\circ} \mathrm{C}$; shaking rate is constant at $\left.100 \mathrm{rpm}\right)$.

\section{Supplementary Files}

This is a list of supplementary files associated with this preprint. Click to download.

- AthabetalJPolymEnviron2022ESI.docx 